Research Paper

\title{
Comparison of Prognostic Indices in NSCLC Patients with Brain Metastases after Radiosurgery
}

\author{
Hong Xiang Gao ${ }^{1,3^{*}}$, Shi Gao Huang ${ }^{2 \bowtie *}$, Jian Fei Du ${ }^{1}$, Xue Cheng Zhang1, Nan Jiang², Wen Xing Kang ${ }^{3}$, Jian \\ $\mathrm{Mao}^{1}$, Qi Zhao ${ }^{2 \bowtie}$ \\ 1. Department of Radiotherapy Oncology, Chang An Hospital, Xi' an, Shaan Xi, China \\ 2. Faculty of Health Sciences, University of Macau, Taipa, Macao SAR, China \\ 3. Radiotherapy Department, 323 Hospital of People's Liberation Army, Xi' an, Shaan Xi, China \\ *These authors equally contributed to the work. \\ $\triangle$ Corresponding authors: Qi Zhao, Faculty of Health Sciences, University of Macau, Avenida da Universidade, Taipa, Macau SAR, China. E-mail: \\ qizhao@umac.mo; or Shigao Huang, E-mail: huangshigao2010@aliyun.com \\ (C) Ivyspring International Publisher. This is an open access article distributed under the terms of the Creative Commons Attribution (CC BY-NC) license \\ (https://creativecommons.org/licenses/by-nc/4.0/). See http://ivyspring.com/terms for full terms and conditions.
}

Received: 2018.07.18; Accepted: 2018.10.05; Published: 2018.11.03

\begin{abstract}
Prognostic indices are commonly used in the context of brain metastases radiotherapy to guide patient decision-making and clinical trial stratification. This study is to choose an appropriate prognostic index (PI) for non-small cell lung cancer (NSCLC) patients with brain metastases (BM) who underwent radiosurgery. A total of 103 patients with BM from NSCLC receiving radiosurgery were analyzed retrospectively. There are six prognostic factors were analyzed, including age, primary tumor control, extracranial metastasis, KPS score, number of lesions, max lesion volume; and four prognostic indices were compared, include Recursive Partitioning Analysis (RPA), Graded Prognostic Assessment (GPA), Score Index for Radiosurgery (SIR), Basic Score for Brain Metastases (BSBM). Survival curves were estimated with the Kaplan-Meier method and compared with a log-rank test stratified according to the Pls. Univariate and multivariate analysis was performed using the Cox regression analysis. The Pl's predictive capacity was compared in terms of Akaike information criterion (AIC), Log-rank $\times 2$, Concordance index ( $\mathrm{C}$-index) and calibration curve. The median survival time was 8 months, and the 6-months and 12 -months survival rate were $61 \%$ and $26 \%$ respectively. All four prognostic indices were correlated with prognosis $(\mathrm{P}<0.005)$. The AIC for BSBM (686.317) was the minimum in the four PIs(range,686.317-739.113). The Log-rank $\times 2$ value for BSBM (77.62) was the maximum in the four Pls (range,23.32-77.62).The C-index for BSBM (0.758)was superior than the other Pls predictive capacity (range,0.611-0.758). The calibration curve showed that the BSBM was able to predict 6-months and 12-months overall survival accurately. In conclusion, the BSBM may be the most accurate prognostic index for patients with BM from NSCLC who underwent radiosurgery.
\end{abstract}

Key words: Non-small cell lung cancer, brain metastases, prognostic index, decision curve analysis, radiosurgery

\section{Introduction}

Lung cancer patients with brain metastases account for $40-50 \%$ of all brain metastases. At the time of the diagnosis of Non-small Cell Lung Cancer (NSCLC), brain metastasis was associated with $10-18 \%$ of patients, and in its course of disease, brain metastases occur in 13-30\%[1]. Moreover, the incidence of brain metastases has been increasing with the aging population and the advancement of imaging technology. Brain metastases cause severe neurologic deficits and cognitive impairment, and their prognosis was poor. For untreated lung cancer patients with brain metastases, median survival time was only 4-11 weeks. Current treatment measures include whole brain radiotherapy (WBRT), stereotactic radiosurgery (SRS), surgery and molecular targeted therapy, but the difference in survival was huge. Only by identifying prognostic factors and separating subgroups of patients, and then choosing appropriate treatment measures can the treatment effect be maximized and the excessive 
medical treatment be avoided. Therefore, a variety of prognostic indices have been established to evaluate the prognosis of patients with brain metastases, so that clinicians can use them as a guide for treatment decision making and for trial eligibility. Recursive Patitioning Analysis (RPA)[2], Graded Prognostic Assessment (GPA)[3], Score Index for Radiosurgery (SIR)[4], Basic Score for Brain Metastases (BSBM)[5] were commonly used in clinical practice.

The RPA was first established in 1997 based on 1,200 patients with brain metastases treated with WBRT in three RTOG studies. RPA was defined as KPS score greater than 70, age less than 65, good primary tumor control and no extracranial metastasis, median survival time was 7.1 months, and such patients were considered as having good prognosis. RPA III was defined as KPS score less than 70, median survival time only 2.3 months, and such patients predicted poor prognosis. The remaining patients were classified into RPA II, with median survival time of 4.2 months.

With the wide application of SRS, Eduardol [4] established SIR in 2000 on the basis of SRS treatment for patients with brain metastases. It was composed of five prognostic factors: age, KPS score, system disease status, number of lesions and the maximum lesion volume. Every prognostic factor is based on different level assign 0 to 2 points, calculated the total points and divided it into three grades, that is, the median survival time of $0-3,4-7$ and $8-10$ as 3 months, 7 months and 33 months.

In 2004, Lorenzoni [5] and his team proposed another simplified prognostic index called BSBM, only 3 prognostic factors were included: KPS score, primary tumor control and extracranial metastasis, assigning 0 or 1 to each prognostic factor at different state, and the total score was 0-3 and divided into four grades with median survival time of 2.6 months, 5.4 months, 11.6 months and 24.6 months.

In 2008, Sperduto[3] established a new prognostic index GPA using data from five RTOG trials. This model was composed of four prognostic factors: age, KPS score, extracranial metastasis and number of lesions. According to the total score of 0-1, 1.5-2.5, 3 and 3.5-4, the survival time was 2.6 months, 3.8 months, 6.9 months and 11 months respectively.

In recent years, the SRS treatment of brain metastasis has become more and more extensive in clinical practice. It was not only effective for oligo metastatic lesions, but also effective for multiple metastatic lesions. Therefore, for the SRS treatment of NSCLC brain metastases, it was greatly significant to select an optimal prognosis index to predict the prognostic. However, these four PIs have different original data sources, all of which contain multiple tumor pathological types. The treatment varies, and RPA was based on patients with WBRT, SIR and BSBM, which were based on patients treated with SRS, while the original GPA data includes WBRT, SRS, and surgery or combined treatment. At present, there was still a controversy about the choice of optimal prognostic index. In this study, the survival and prognostic factors of patients with NSCLC brain metastases after SRS treatment were analyzed, and the optimal prognostic index was obtained by comparing the four existing PIs statistically.

\section{Methods and materials}

\section{Database Composition}

Between January 2010 and December 2014, 103 patients underwent Gamma knife radiosurgery for NSCLC brain metastases at the 323 Hospital of People's Liberation Army were included in study. Patients with missed follow-up were excluded from the study. All included patients'age were ranged from 33 to 81 years which included male 59(57\%) and female $44(43 \%)$. They were confirmed diagnosed by bronchoscopic biopsy and head enhanced 1.5T magnetic resonance imaging (MRI) (SIEMENS Symphony, Germany). The institutional ethics approval was obtained for this data base analysis. The patients' characteristics and demographics were shown in the Table 1.

Table 1. Patients characteristics and demographics

\begin{tabular}{lll}
\hline Characteristics & $\mathrm{N}(\%)$ \\
\hline $\begin{array}{l}\text { Patients } \\
\text { Gender }\end{array}$ & & 103 \\
Male & & \\
Female & & $59(57 \%)$ \\
Age(years) & Median & $44(43 \%)$ \\
& Range & 58 \\
KPS & Median & $48(33-81)$ \\
& Range & 70 \\
Extracranial matastasis & No & $30(60-90)$ \\
& Yes & $66(64.1 \%)$ \\
Primary tumor control & No & $37(35.9 \%)$ \\
& Yes & $59(57.3 \%)$ \\
Number of lesions & Median & $44(42.7 \%)$ \\
& Range & 2 \\
& Total & $5(1-6)$ \\
Max lesion volume(ml) & Median & 207 \\
& Range & 3.4 \\
\hline
\end{tabular}

\section{Treatment}

Using gamma-knife (Masep SRRS, Shenzhen, China) to perform radiosurgery, with MRI head enhancement scanning. The patients were rigidly fixed with the LEKSELL stereotactic headframe. The target area was defined as the MRI enhancement area which expands $1-2 \mathrm{~mm}$. The prescription dose in the target area was given in accordance with the NCCN 
guideline recommendation scheme (the maximum prescribed dose was 24Gy, 18Gy, 15Gy in accordance with the maximum diameter of $20 \mathrm{~mm}, 21-30 \mathrm{~mm}$, $31-40 \mathrm{~mm}$ respectively). Of the 103 patients, only 4 had ever received molecular targeted therapy after radiotherapy.

\section{Follow-up}

The patients were checked by blood test, computed tomography or PET/CT scan of the chest, abdomen, and cervical region at least once a year. Survival status was evaluated by the physician.

\section{Statistics Analysis}

The statistical analysis was performed using SPSS software (version 19.0, IBM, American) and R software (version 3.5.1). Statistical significance was assumed at a two-sided probability value $\mathrm{p}<0.05$. All dates were represented by Mean $\pm \mathrm{SD}$, Multivariate analysis was performed by the Cox proportional hazard model. Overall survival (OS) was defined from the date of radiosurgery to the date of death. The survival rate was calculated by using the Kaplan-Meier method, and values were compared by using the Log-rank test. Use the Cox regression analysis by the enter procedure for prognostic factors selection. The PI's predictive capacity was compared in terms of its discrimination and calibration. Discrimination refers to the ability to distinguish high-risk patients from low-risk patients and is commonly quantified via a concordance index which measures the level of concordance between the order of predicted probabilities and the order of the events of interest. One such index is Harrell's c index which, for survival data, is defined as the proportion of all pairs of subjects whose survival time can be ordered such that the subject with the higher predicted survival is the one who survived longer. Calibration refers to whether the predicted probabilities agree with the observed probabilities and are usually assessed using calibration plots. Therefore, the Akaike information criterion (AIC), Concordance index (C-index) and Log-rank $\chi 2$ were used to determine its discrimination, and the calibration curve were used to determine its calibration. The AIC, Log-rank $X^{2}$ and C-index were calculated by using the survival analysis function package of $\mathrm{R}$ software. A smaller AIC value indicated a more goodness-of-fit, and a larger C-index value indicated a better predicted precision of outcome. The Log-rank $\chi^{2}$ value indicated the difference of survival distribution among different classes of the four PIs respectively. The greater the value, the greater the difference in survival may be. Calibration curve visually evaluates the consistency between the predicted value and the actual value of the PIs.

\section{Results}

\section{Patients' survival}

At the end of follow-up, all 103 patients were dead which were included in this study and the missed follow-up patients were excluded. The mean survival time was 10.262 months (95\% CI 8.861-11.663 months), and median survival time (MST) was 8 months (95\% CI 6.708-9.292 months). The 6-month and the 1-year survival rate was $61 \%$ and $26 \%$, respectively. The survival results classified by prognostic indices were shown in Table 2. The survival curve of each prognostic index was obtained by K-M survival analysis (Fig 1).

Table 2. Prognostic indices survival

\begin{tabular}{|c|c|c|c|c|c|c|}
\hline PI & Classes & n (\%) & 6-months survival rate & 12-months survival rate & Mean survival time (months) & Median survival time (months) \\
\hline RPA & \multicolumn{6}{|c|}{$x^{2}=69.468, p=0.000$} \\
\hline & I & $14(14 \%)$ & 0.909 & 0.786 & 21.143 & 20 \\
\hline & II & $74(72 \%)$ & 0.649 & 0.216 & 9.500 & 8 \\
\hline & III & $15(14 \%)$ & 0.133 & 0 & 3.867 & 4 \\
\hline SIR & \multicolumn{6}{|c|}{$\chi^{2}=24.427, p=0.000$} \\
\hline & $1-3$ & $11(11 \%)$ & 0.182 & N & 5 & 5 \\
\hline & $4-7$ & $82(80 \%)$ & 0.622 & 0.232 & 9.732 & 8 \\
\hline & $8-10$ & $10(9 \%)$ & 1 & 0.744 & 20.4 & 19 \\
\hline GPA & \multicolumn{6}{|c|}{$\chi^{2}=30.621, p=0.000$} \\
\hline & $0-1$ & $12(12 \%)$ & 0.333 & 0 & 5.250 & 6 \\
\hline & $1.5-2.5$ & $69(67 \%)$ & 0.536 & 0.174 & 8.812 & 7 \\
\hline & 3 & $15(14 \%)$ & 1 & 0.600 & 16.667 & 14 \\
\hline & $3.5-4$ & $7(7 \%)$ & 1 & 0.821 & 19.429 & 20 \\
\hline BSBM & \multicolumn{6}{|c|}{$\chi^{2}=75.481, p=0.000$} \\
\hline & 0 & $15(14 \%)$ & 0.200 & 0 & 4.600 & 5 \\
\hline & 1 & $40 \quad(39 \%)$ & 0.475 & 0.025 & 6.675 & 6 \\
\hline & 2 & $30(29 \%)$ & 0.800 & 0.367 & 11.500 & 12 \\
\hline & 3 & $18(18 \%)$ & 0.936 & 0.833 & 20.889 & 20 \\
\hline
\end{tabular}

PI: Prognostic Index; RPA:Recursive Patitioning Analysis; SIR: Score Index for Radiosurgery; GPA: Graded Prognostic Assessment; BSBM: Basic Score for Brain Metastases. 

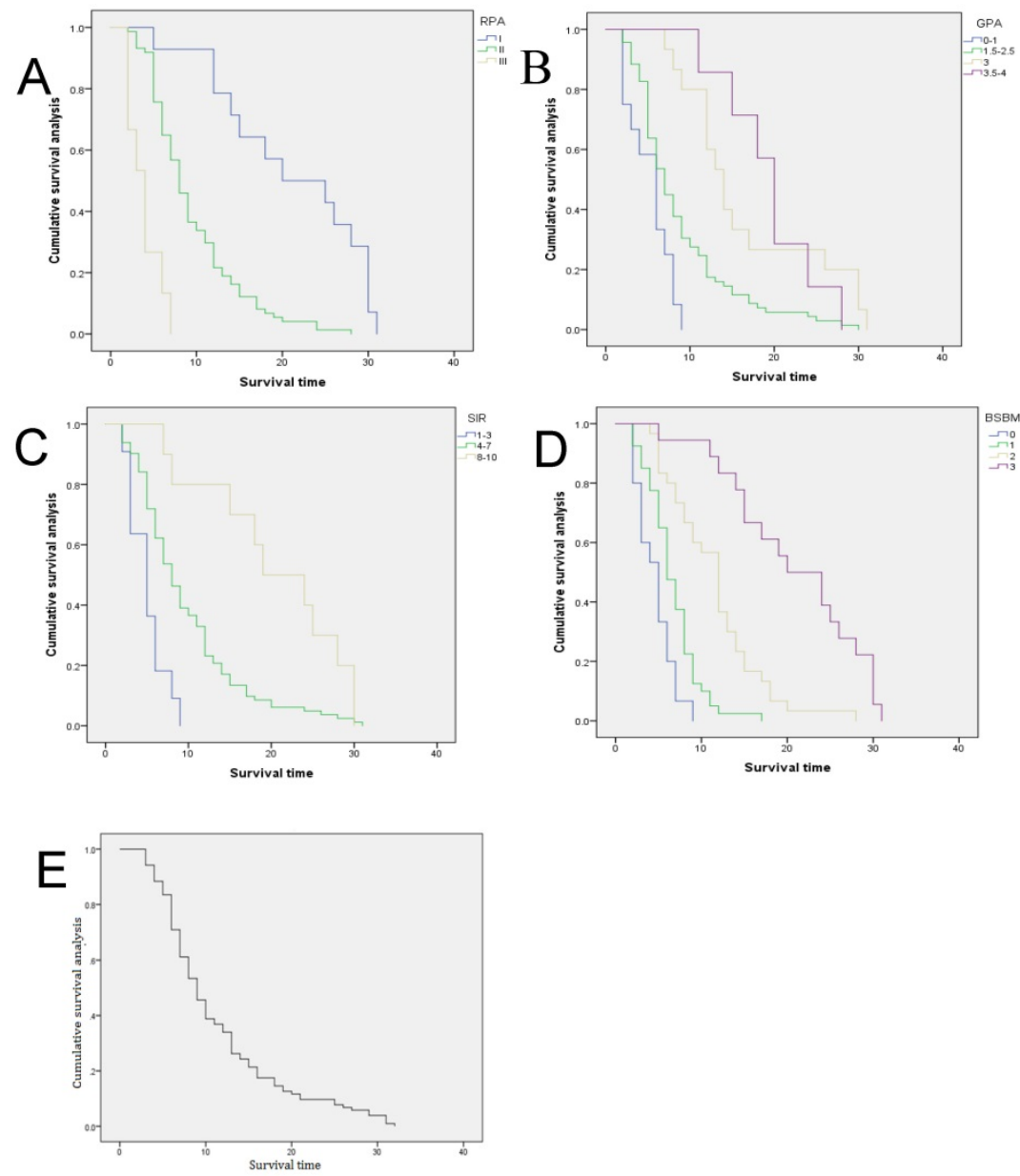

Figure 1. Overall survival curve for the four Prognostic Indices, (A) RPA, Recursive Partitioning Analysis, (B) GPA, Graded Prognostic Assessment,(C) SIR, Score Index for Radiosurgery,(D) BSBM, Basic Score for Brain Metastases and Overall survival curve for all patients (E).

\section{Prognostic factors}

Univariable and multivariable analysis was performed using Cox regression analysis for the six prognostic factors constituting the PIs. As shown in Table 3, KPS (adjusted HR 0.916; 95\% CI,0.889-0.944), extracranial metastasis (adjusted HR 2.296;95\% CI,1.407-3.749), primary tumor control (adjusted HR $0.444 ; 95 \% \quad \mathrm{CI}, 0.285-0.693) \quad$ were independent prognostic factors for OS.

Table 3. Univariable and multivariable analysis for prognostic factors

\begin{tabular}{lllll}
\hline & \multicolumn{2}{l}{ Univariable Analysis } & \multicolumn{2}{l}{ Multivariable Analysis } \\
\hline Prognostic factors & $\mathrm{p}$ & $\mathrm{HR}(95 \% \mathrm{CI})$ & $\mathrm{p}$ & $\mathrm{HR}(95 \% \mathrm{CI})$ \\
\hline Age & 0.721 & $1.004(0.984-1.024)$ & 0.509 & $1.006(0.988-1.024)$ \\
KPS & 0.000 & $0.904(0.878-0.931)$ & 0.000 & $0.916(0.889-0.944)$ \\
$\begin{array}{l}\text { Extracranial } \\
\text { metastasis }\end{array}$ & 0.000 & $3.341(2.131-5.238)$ & 0.001 & $2.296(1.407-3.749)$ \\
$\begin{array}{l}\text { Primary tumor } \\
\text { control }\end{array}$ & 0.001 & $0.477(0.312-0.729)$ & 0.000 & $0.444(0.285-0.693)$ \\
$\begin{array}{l}\text { Number of lesions } \\
\text { Max lesion volume }\end{array}$ & 0.002 & $1.277(1.091-1.494)$ & 0.136 & $1.135(0.961-1.340)$ \\
\hline
\end{tabular}

\section{Prognostic indices}

COX regression analysis was conducted for RPA, GPA, SIR and BSBM, respectively, as showed in the Table 4. All four PIs could predict survival, and there were significant differences $(\mathrm{P}<0.05)$ among classes of PIs.

Table 4. Multivariable Cox Regression analysis for prognostic indices

\begin{tabular}{llllll}
\hline PI & Classes & Wald & P & HR & $95 \% \mathrm{CI}$ \\
\hline RPA & & 49.361 & 0.000 & & \\
& I vs. II & 17.867 & 0.000 & 4.629 & $2.275-9.419$ \\
& II vs. III & 47.804 & 0.000 & 26.155 & $10.368-65.976$ \\
SIR & & 19.446 & 0.000 & & \\
& 1-3 vs.4-7 & 10.470 & 0.001 & 0.335 & $0.172-0.649$ \\
& $4-7$ vs.8-10 & 19.331 & 0.000 & 0.127 & $0.051-0.319$ \\
GPA & & 24.598 & 0.000 & & \\
& $0-1$ vs. $1.5-2.5$ & 6.672 & 0.010 & 0.431 & $0.227-0.816$ \\
& $1.5-2.5$ vs.3 & 18.955 & 0.000 & 0.159 & $0.069-0.364$ \\
& 3 vs. $3.5-4$ & 13.933 & 0.000 & 0.154 & $0.058-0.411$ \\
BSBM & & 56.692 & 0.000 & & \\
& 0 vs.1 & 5.959 & 0.015 & 0.467 & $0.253-0.861$ \\
& 1 vs.2 & 27.940 & 0.000 & 0.146 & $0.071-0.298$ \\
& 2 vs.3 & 49.232 & 0.000 & 0.040 & $0.017-0.099$ \\
\hline
\end{tabular}



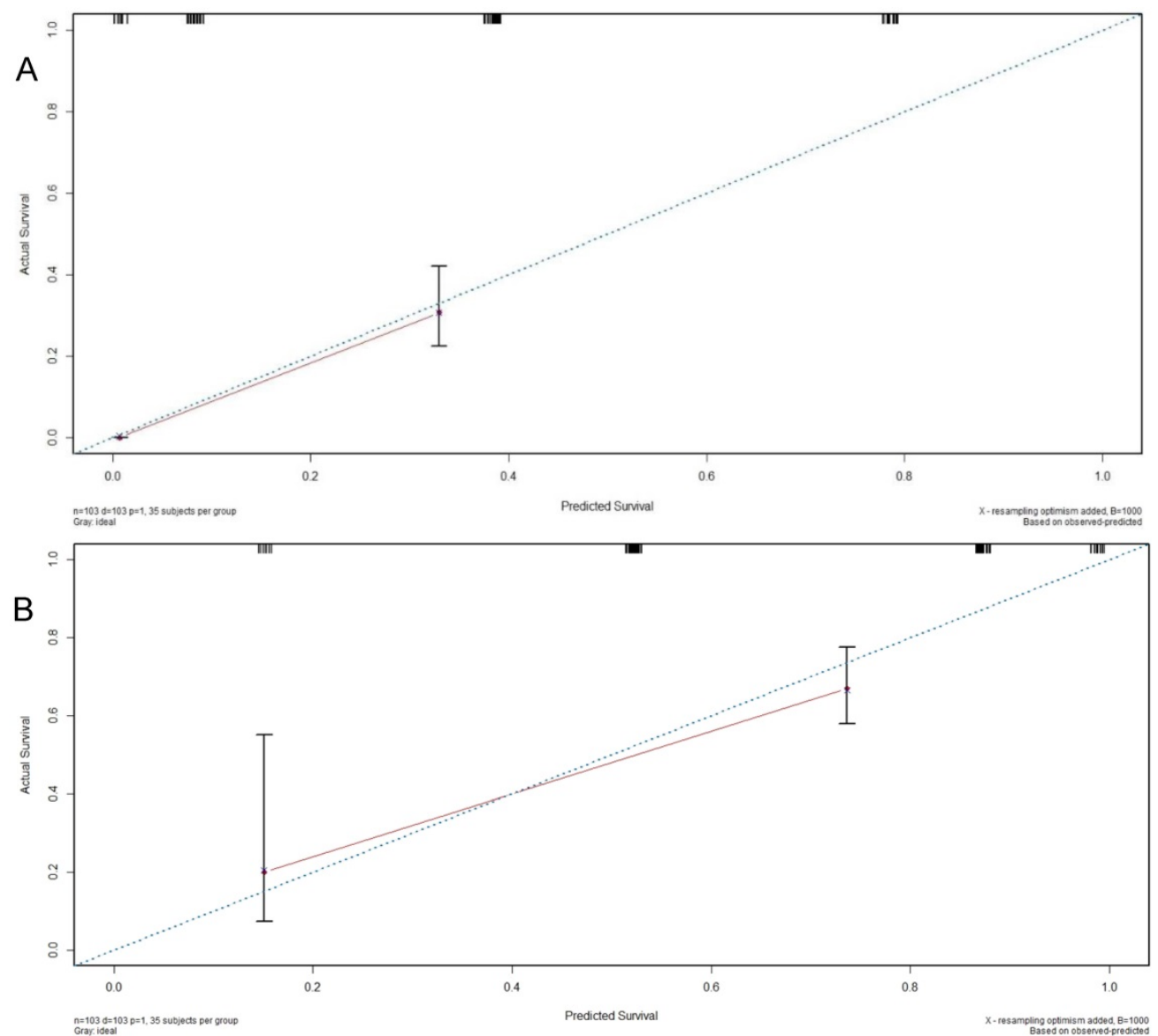

Figure 2. Calibration cure for the prediction of 12-months OS (A) and 6-months OS (B) by BSBM. BSBM, Basic Score for Brain Metastases; OS , overall survival.

The comparison among PIs was performed by the Log-rank $\chi 2$, AIC, and C-index. As shown in Table 5, the AIC value for the BSBM (686.317) was smallest when compared with the others (RPA, 703.873; GPA,734.531; SIR,739.113), which indicated that the BSBM prognostic index make a better prognostic discrimination. In addition, the calibration was demonstrated by calibration curve prediction of 6 -months OS and 12-months OS (Fig 2) by BSBM index. All the statistical indicators showed that BSBM was the optimal PI.

Table 5. Comparison among four Pls in Multivariate Cox Regression Analysis

\begin{tabular}{llll}
\hline PI & Log-rank $\chi^{2}$ & AIC value & C-index $(95 \% \mathrm{CI})$ \\
\hline RPA & 70.59 & 703.873 & $0.682(0.627-0.737)$ \\
GPA & 27.99 & 734.531 & $0.655(0.592-0.718)$ \\
SIR & 23.32 & 739.113 & $0.611(0.560-0.662)$ \\
BSBM & 77.62 & 686.317 & $0.758(0.689-0.827)$ \\
\hline
\end{tabular}

\section{Discussion}

There were 7 prognostic indices for brain metastases in the published literature [6-12], among which RPA, GPA, SIR and BSBM were most commonly used. In 1997, Gaspar established RPA, whose results were verified by the same author in 2000[13], including 445 cases, the median survival time of RPA I was 6.2 months, and that of RPA II was 3.8 months, having no difference from the original data. Since then, several authors have confirmed its usability[14-17]. However, in these validation studies, the survival time of patients treated with WBRT was consistent with the original data, while the survival time of patients treated with SRS was different, which restricted the application of RPA in SRS. Another limitation is the RPA IIthat contains the larger proportion of cases, and it is difficult to accurately assess prognosis because of the heterogeneity. The number of cases in this study of RPA II was 74, accounting for $72 \%$ of the total. Moreover, RPA III only by KPS is less than 70 for classification basis, and this may be caused by various causes, including neurological symptoms caused by brain metastatic tumor invasion, the progress of the extracranial other lesions, cancerous pain, pathologic fracture, pneumonia, anemia caused by chemotherapy or other nonneoplastic lesion, not all of which were cancer related.

With the wide application of SRS, the number of 
lesions has been proved to be another independent factor in predicting prognosis[18]. SIR scoring was established, which was derived from a limited number of patients treated with SRS. However, several groups confirmed the performance of the SIR in patients treated with SRS, surgery and WBRT, and some of them were with large number of patients[3, 19]. When evaluating the SIR and RPA indices in their SRS database, the group from Belgium, arrived at BSBM, which was composed of just three factors, simple to use, and has been proved effective by many studies.

In 2008, Sperduto [3] found some limitations by analyzing the previous three prognostic indices:1) The number of lesions was not included in the RPA and BSBM models, and the RTOG 9508 study[20] clearly indicated that the number of lesions was an independent factor affecting the prognosis.2)The previous PIs required an assessment of the systemic conditions, however, this kind of evaluation varies with different imaging technology and examination time.3)SIR included treatment-related prognostic factors(max lesion volume)before evaluating the prognosis, and the significance of the prognostic index was to make optimal therapeutic choices after evaluating the prognosis. Therefore, they established GPA and compared its predictive ability with RPA, SIR and BSBM by statistical methods. The results showed that GPA and RPA had the same predictive ability and were superior to other prognostic indices. But the GPA is more objective, easier to quantify and easier to use. Then, other researchers confirmed its reliability [21-24].

KPS score and extracranial metastasis were included in these prognostic indices, and the results of this study also confirmed that they were independent prognostic factors. However, the KPS score was used to evaluate the physical condition of patients, and the causes of such symptoms and signs were not distinguished, resulting in the evaluation of variability and inaccuracy. Such as patients with nerve dysfunction, the KPS score was low. However, this kind of functional disorder is mostly caused by edema, which was reversible and can be recovered within a short period of time after the application of dehydration and hormone therapy, thus resulting in the misjudgment of KPS score. The evaluation of extracranial metastasis was closely related to the development and application of imaging technology. For example, the detection rate of metastatic tumors by CT was significantly lower than that of PET/CT.

Generally, age was considered as an adverse prognostic factor in patients with brain metastases, and age was also a component of RPA, GPA and SIR, but some authors questioned the value of age as a prognostic factor. Watanabe[25] retrospectively analyzed 2552 patients with brain metastatic tumor treated with gamma knife, and found that SRS still benefited over 65 years old. There was no statistically significant difference in median survival between groups aged 65 to 79 and those aged over 80, and there was no difference in neurologic death or SRS related complications. In this study, age was used as a continuous variable or was stratified at 65 years old for univariate and multivariate analysis, and the results showed no significant correlation with survival. It may be that the age distribution in this group is mainly below 65 years old, and the median age is 58 years old.

It was traditionally believed that patients with multiple brain metastases have micrometastases throughout the brain, but the current imaging techniques cannot detect it, and the survival time of such patients was often very short. In 2010, Chang[26] divided 323 patients with brain metastatic tumor treated with gamma knife into four groups according to the number of lesions:1-5 lesions, 6-10 lesions, 11-15 lesions, and more than 15 lesions. The results showed that there was no significant difference in the survival of each group. Other authors have also verified this result with a large amount of data[27, 28]. However, the number of lesions ultimately affects the tumor burden, and the maximum lesion volume was only part of the tumor burden, some studies[29] have shown that the total tumor volume was ultimately an important prognostic factor. In this study, the median number of lesions was 2, and the median volume of the largest lesions was $3.4 \mathrm{ml}$, neither of which showed a significant correlation with survival. This may be due to the selection of patients in this study before treatment in order to reduce the possibility of complications such as edema after treatment. Clinicians had empirically rejected patients with large lesion volume or heavy tumor burden as treatment indications.

Survival analysis was the main method in most studies of prognosis indices comparison. As long as there were significant differences in the results of multivariate analysis, the best PI can be obtained. However, univariate and multivariate COX regression analysis was carried out for the four PIs in this study, the results showed that all of them could predict survival, and there were significant differences among subgroups of each PI. In statistics, the predictive ability of a prognostic index was evaluated not only by its predictive survival, but also by its discrimination and calibration. All the statistical indicators in this study showed that BSBM was the optimal PI. 
With the development of molecular biology and gene detection technology's widely use, molecular targeted therapy and immunotherapy more and more applied in the patients with brain metastases, such as VEGF, EGFR, ALK and its Her-2 as a new type of prognostic factors, and researchers had established a new prognostic model accordingly [12].The disadvantage of this study was that the retrospective analysis did not include these new prognostic factors, which may lead to the deficiency in prognostic indices comparison.

In conclusion, the four prognostic indices of RPA, GPA, SIR, BSBM were associated with survival and could predict clinical prognosis for NSCLC patients with $\mathrm{BM}$ who underwent radiosurgery and the BSBM may be the most accurate prognostic index.

\section{Acknowledgements}

This work was supported by the Science and Technology Development Fund of Macau (FDCT/131/2016/A3, FDCT/0015/2018/A1), the Guangzhou Science and Technology Program (201807010004), Start-up Research Grand (SRG2016-00082-FHS) and the intramural research program of Faculty of Health Sciences, University of Macau, and National Natural Science Foundation of China (31440041).

\section{Abbreviations}

PI: Prognostic Index; BM: Brain Metastases; NSCLC: Non-small Cell Lung Cancer; RPA: Recursive Partitioning Analysis; SIR: Score Index for Radiosurgery; GPA: Graded Prognostic Assessment; BSBM: Basic Score for Brain Metastases; AIC: Akaike information criterion; C-index: Concordance index; MST: Median Survival Time; WBRT: Whole Brain Radiotherapy; SRS: Stereotactic Radiosurgery; MRI: Magnetic Resonance Imaging; OS: Overall Survival;K-M: Kaplan-Meier; KPS: Karnofsky Performance Status.

\section{Competing Interests}

The authors have declared that no competing interest exists.

\section{References}

1. Taillibert S, Le Rhun É. Epidemiology of brain metastases. Cancer radiotherapie: journal de la Societe francaise de radiotherapie oncologique. 2015; 19: 3-9.

2. Gaspar L, Scott C, Rotman M, Asbell S, Phillips T, Wasserman T, et al. Recursive partitioning analysis (RPA) of prognostic factors in three Radiation Therapy Oncology Group (RTOG) brain metastases trials. International Journal of Radiation Oncology* Biology* Physics. 1997; 37: 745-51.

3. Sperduto PW, Berkey B, Gaspar LE, Mehta M, Curran W. A new prognostic index and comparison to three other indices for patients with brain metastases: an analysis of 1,960 patients in the RTOG database. International Journal of Radiation Oncology* Biology* Physics. 2008; 70: 510-4.

4. Weltman E, Salvajoli JV, Brandt RA, de Morais Hanriot R, Prisco FE, Cruz JC, et al. Radiosurgery for brain metastases: a score index for predicting prognosis. International Journal of Radiation Oncology* Biology* Physics. 2000; 46: 1155-61.

5. Lorenzoni J, Devriendt D, Massager N, David P, Ruíz S, Vanderlinden B, et al. Radiosurgery for treatment of brain metastases: estimation of patient eligibility using three stratification systems. International Journal of Radiation Oncology* Biology* Physics. 2004; 60: 218-24.

6. Gaspar L, Scott C, Rotman M, Asbell S, Phillips T, Wasserman T, et al. Recursive partitioning analysis (RPA) of prognostic factors in three Radiation Therapy Oncology Group (RTOG) brain metastases trials. International journal of radiation oncology, biology, physics. 1997; 37: 745-51.

7. Lagerwaard FJ, Levendag PC, Nowak PJ, Eijkenboom WM, Hanssens PE, Schmitz PI. Identification of prognostic factors in patients with brain metastases: a review of 1292 patients. International journal of radiation oncology, biology, physics. 1999; 43: 795-803.

8. Weltman E, Salvajoli JV, Brandt RA, de Morais Hanriot R, Prisco FE, Cruz JC, et al. Radiosurgery for brain metastases: a score index for predicting prognosis. International journal of radiation oncology, biology, physics. 2000; 46: 1155-61.

9. Lorenzoni J, Devriendt D, Massager N, David P, Ruiz S, Vanderlinden B, et al. Radiosurgery for treatment of brain metastases: estimation of patient eligibility using three stratification systems. International journal of radiation oncology, biology, physics. 2004; 60: 218-24.

10. Sperduto PW, Berkey B, Gaspar LE, Mehta M, Curran W. A new prognostic index and comparison to three other indices for patients with brain metastases: an analysis of 1,960 patients in the RTOG database. International journal of radiation oncology, biology, physics. 2008; 70: 510-4.

11. Rades D, Dunst J, Schild SE. A new scoring system to predicting the survival of patients treated with whole-brain radiotherapy for brain metastases. Strahlentherapie und Onkologie : Organ der Deutschen Rontgengesellschaft. 2008; 184: 251-5.

12. Sperduto PW, Yang TJ, Beal K, Pan H, Brown PD, Bangdiwala A, et al. Estimating Survival in Patients With Lung Cancer and Brain Metastases: An Update of the Graded Prognostic Assessment for Lung Cancer Using Molecular Markers (Lung-molGPA). JAMA oncology. 2017; 3: 827-31.

13. Gaspar LE, Scott C, Murray K, Curran W. Validation of the RTOG recursive partitioning analysis (RPA) classification for brain metastases. International Journal of Radiation Oncology* Biology* Physics. 2000; 47: 1001-6.

14. Agboola O, Benoit B, Cross P, Da Silva V, Esche B, Lesiuk H, et al. Prognostic factors derived from recursive partition analysis (RPA) of Radiation Therapy Oncology Group (RTOG) brain metastases trials applied to surgically resected and irradiated brain metastatic cases. International Journal of Radiation Oncology* Biology* Physics. 1998; 42: 155-9.

15. Nieder C, Nestle U, Motaref B, Walter K, Niewald M, Schnabel K. Prognostic factors in brain metastases: should patients be selected for aggressive treatment according to recursive partitioning analysis (RPA) classes? International Journal of Radiation Oncologybiologyphysics. 2000; 46: 297-302.

16. Chidel MA, Suh JH, Reddy CA, Chao ST, Lundbeck MF, Barnett GH. Application of recursive partitioning analysis and evaluation of the use of whole brain radiation among patients treated with stereotactic radiosurgery for newly diagnosed brain metastases. International Journal of Radiation Oncology biology physics. 2000; 47: 993-9.

17. Tendulkar RD, Liu SW. RPA classification has prognostic significance for surgically resected single brain metastasis. International Journal of Radiation Oncology Biology Physics. 2006; 66: 810-7.

18. Weltman E, Salvajoli JV, e Oliveira VC, Brandt RA, da Cruz JC, de Oliveira Borges SR, et al. Score index for stereotactic radiosurgery of brain metastases. Journal of Radiosurgery. 1998; 1: 89-97.

19. Villà S, Weber DC, Moretones C, Mañes A, Combescure C, Jové J, et al. Validation of the new Graded Prognostic Assessment scale for brain metastases: a multicenter prospective study. Radiation Oncology. 2011; 6: 1 .

20. DW A, CB S, PW S, AE F, LE G, MC S, et al. Whole brain radiation therapy with or without stereotactic radiosurgery boost for patients with one to three brain metastases: phase III results of the RTOG 9508 randomised trial. Lancet. 2004; 363: 1665-72.

21. Sperduto P, Sneed P, Bhatt A, Schwer A, Fiveash J, Chiang V, et al. A multi-institutional validation study of a new prognostic index (graded prognostic assessment, GPA) for patients with brain metastases. International Journal of Radiation Oncology* Biology* Physics. 2008; 72: S51.

22. Sperduto CM, Watanabe Y, Mullan J, Hood T, Dyste G, Watts C, et al. A validation study of a new prognostic index for patients with brain metastases: the Graded Prognostic Assessment. 2008.

23. Sperduto PW, Kased N, Roberge D, Xu Z, Shanley R, Luo X, et al. Summary report on the graded prognostic assessment: an accurate and facile diagnosis-specific tool to estimate survival for patients with brain metastases. Journal of Clinical Oncology. 2012 Feb 1; 30(4): 419-425.

24. Luo J, Zhu H, Tang $\mathrm{Y}$, Wang $\mathrm{H}$, Zhou $\mathrm{X}$, Lu X, et al. Analysis of prognostic factors and comparison of prognostic index scores in patients with brain metastases after whole-brain radiotherapy. International Journal of Clinical \& Experimental Medicine. 2014; 7: 5217-25.

25. Watanabe S, Yamamoto M, Sato Y, Kawabe T, Higuchi Y, Kasuya H, et al. Stereotactic radiosurgery for brain metastases: a case-matched study comparing treatment results for patients 80 years of age or older versus patients 65-79 years of age Clinical article. Journal of Neurosurgery. 2014; 121: 1148-57. 
26. Chang WS, Kim HY, Chang JW, Park YG, Chang JH. Analysis of radiosurgical results in patients with brain metastases according to the number of brain lesions: is stereotactic radiosurgery effective for multiple brain metastases? Journal of Neurosurgery. 2010; 113 Suppl: 73-8.

27. MA K, EK O, AI Y, I P, J S, B C, et al. Survival of Patients With Multiple Intracranial Metastases Treated With Stereotactic Radiosurgery: Does the Number of Tumors Matter? American journal of clinical oncology. 2016.

28. Yamamoto M, Serizawa T, Shuto T, Akabane A, Higuchi Y, Kawagishi J, et al. Stereotactic radiosurgery for patients with multiple brain metastases (JLGK0901): a multi-institutional prospective observational study. Lancet Oncology. 2014; 15: 387-95.

29. Routman DM, Bian SX, Diao K. The growing importance of lesion volume as a prognostic factor in patients with multiple brain metastases treated with stereotactic radiosurgery. Cancer Medicine . 2018; 7: 757-64. doi:10.1002/ cam4.1352. 